\title{
Infections in breast implants: a review with a focus on developing countries
}

\author{
Corrado Rubino ${ }^{1}$, Sergio Brongo ${ }^{1}$, Domenico Pagliara ${ }^{1}$, Roberto Cuomo ${ }^{1}$, Giulia Abbinante ${ }^{1}$, Nicola \\ Campitiello $^{1}$, Fabio Santanelli ${ }^{2}$, Daniela Chessa ${ }^{3}$ \\ ${ }^{1}$ Department of Medicine and Surgery, Plastic Surgery Unit, Azienda Ospedaliera Universitaria OO.RR. San \\ Giovanni di Dio e Ruggi d'Aragona, University of Salerno, Salerno, Italy \\ ${ }^{2}$ Department of Medicine and Surgery, Plastic Surgery Unit, Sant'Andrea Hospital, School of Medicine and \\ Psychology, Sapienza University of Rome, Rome, Italy \\ ${ }^{3}$ Department of Biomedical Science, School of Medicine, University of Sassari, Sassari, Italy
}

\begin{abstract}
The risk of surgical site infection is always present in surgery; the use of prosthetic materials is linked to an increased possibility of infection. Breast augmentation and breast reconstruction with implants are gaining popularity in developing countries. Implant infection is the main complication related to breast aesthetic and reconstructive surgery. In the present paper, we reviewed the current microbiological knowledge about implant infections, with particular attention to risk factors, diagnosis, clinical management, and antibiotic prophylaxis, focusing on reports from developing countries. After breast aesthetic surgery, up to $2.9 \%$ of patients develop a surgical site infection, with an incidence of $1.7 \%$ for acute infections and $0.8 \%$ for late infections. The rate of surgical site infection after post-mastectomy breast reconstruction is usually higher, ranging from $1 \%$ to $53 \%$. The clinical features are not constant, and bacterial culture with antibiogram is the gold standard for diagnosis and for identification of antibiotic resistance. While waiting for culture results, empiric therapy with vancomycin and extendedspectrum penicillins or cephalosporins is recommended. Some patients require removal of the infected prosthesis. The main methods to bring down the risk of infection are strict asepsis protocol, preoperative antibiotic prophylaxis, and irrigation of the surgical pocket and implant with an antibiotic solution.
\end{abstract}

Key words: breast implant infections; acute infections; late infections; fungal infections; rare infections.

J Infect Dev Ctries 2014; 8(9):1089-1095. doi:10.3855/jidc.3898

(Received 19 July 2013 - Accepted 08 February 2014)

Copyright (C) 2014 Rubino et al. This is an open-access article distributed under the Creative Commons Attribution License, which permits unrestricted use, distribution, and reproduction in any medium, provided the original work is properly cited.

\section{Epidemiology}

Implant infection is the main complication related to breast aesthetic and reconstructive surgery [1]. After breast aesthetic augmentation, up to $2.9 \%$ of women are affected by infection [2], with an incidence of $1.7 \%$ for acute infections versus an incidence of $0.8 \%$ for late infections, as confirmed by retrospective cohort studies with long-term follow-ups [1]. In developing countries, this problem is even more prevalent; surgery has a $50 \%$ rate of possible complications, of which infection is the main complication [3]. The rate of surgical site infection after post-mastectomy breast reconstruction is usually higher, ranging from $1 \%$ to $53 \%[4,5]$. In breast reconstruction, a higher rate of infection was reported with expander implants compared to reconstruction with autologous tissue such as latissimus dorsi flaps [6]. The highest rates were found in women undergoing immediate reconstruction [4]. The reported number of infections after mammary augmentation and reconstruction is lower than the real incidence, because of the lack of a surveillance network of patients based on long-time follow-ups. The economic commitment for management of implant infections is significant. Kirkland et al. [7] reported an increase of hospital permanence for surgical site infection of 6.5 days, with related health care costs. In the United States, health care costs for surgical site infection after breast surgery were estimated to be about $\$ 4,091$ [7], or $\$ 574$ in the outpatient setting [9]. In developing countries, the risk of complications of surgery including infection - is the second most frequent reason for women rejecting immediate breast reconstruction after mastectomy [10].

\section{Risk factors}

Several risk factors associated with an increased rate of implant infections have been studied. For convenience, it is useful to separate pre-operative, intra-operative, and post-operative risk factors. 
Clinical patient conditions and surgical technique are the most important elements in setting the overall risk of surgical site infection. Within the preoperative risk factors, breast size larger than $\mathrm{C}$ cup, body mass index greater than 30, smoking, diabetes, prior breast surgery, chest wall radiation therapy, and neoadjuvant chemotherapy have been reported $[6,11,12]$. However, as found by a recent retrospective study on the statistical significance of risk factors, increased body mass index, diabetes, tobacco use, and chemotherapy do not represent independent risk factors with statistical significance [11]. Intraoperatively, the surgical technique seems to be able to influence the infection rate, as demonstrated by the higher number of infective complications associated with periareolar or transareolar approaches, probably due to contamination of the implant by endogenous flora of the nipple or breast ducts. Consequentially, adequate skin asepsis can reduce the risk of surgical site infections. Axillary node dissection represents a risk factor able to increase the chance of implant infection by 6.29 times [12]. Reconstructive surgery shows a greater risk than does breast augmentation, probably due to the higher level of ischemia, scarring, and skin atrophy resulting from a longer or repeated surgical engagement of the thoraco-mammary area. Surgical site infection seems to be more frequent in immediate reconstruction rather than in delayed and multi-step reconstruction; this finding is probably linked to the greater possibility of surgical bed contamination in the one-step approach. According to Araco et al. [13], the drains placement could be associated with a fivefold increased risk of surgical site infections; however, McCarthy et al. [14] found no differences in infection risk in patients who did and patients who did not have drains during surgery.. Francis et al. [11] suggested that it could be the lack of drain placement that increases the probability of infection, although only moderately. Several features of implants, such as texture or polyurethane coating [15], do not seem to be able to modify the infection rate. Instead, implant coating with acellular dermal matrix increases the risk of infection, as confirmed by Liu et al. [16] in a case report of 470 patients that noted, after immediate reconstruction, a $4.2 \%$ rate of implant removal due to infection for implants coated with acellular dermal matrix, compared to $2.4 \%$ for non-coated implants. With regard to contamination of saline implants, although the shell is not permeable to bacteria [17], bacteria can enter into the lumen through the implant valve [18]. It is yet to be confirmed whether there are differences in overall infection rates between silicone implants and saline implants. Postoperatively, the formation of a seroma or hematoma [17] may represent an appropriate pabulum for bacterial overgrowth. Other possible predisposing factors include adjuvant chemotherapy, breast trauma, nipple piercing [19], and all diagnostic and therapeutic procedures able to detect bacteremia, even if transient. It is worth noting the possibility of hematogenous dissemination from a remote focus of infection, with secondary colonization of the implant surface [15]. Peled et al. found that, after mastectomy and immediate reconstruction, adjuvant chemotherapy was associated with a higher overall rate of infection (44\%) compared to neoadjuvant chemotherapy (23\%) and lack of chemotherapy (25\%) [20]. Adhesive bandage of the surgical dressing can cause severe contact dermatitis with subsequent superinfection of the skin and depth diffusion in the implant. However, an accurate evaluation of risk factors based on prospective studies with long-term follow-ups is not yet available.

As a solution for developing countries with small budgets for health care spending, Agrawal suggested the reuse of expanders and stressed that, in his experience, the complication rate is similar for reused or fresh expanders [21]. However, the reuse of expanders in developing countries is not recommended, as reports have demonstrated an increased incidence of complications [22] and postimplantation development of clinical symptomatology with fatigue, fever, shortness of breath, and pleural effusion, compatible with infection [23]. According to Anger [22], the higher number of complications associated with the reused expander could lead to greater health care spending than would the purchase of a new expander.

\section{Acute infections}

Acute infections represent the most common postoperative infections and occur more frequently than late infections, usually between the first and the sixth week after surgery [15]. The average onset time is 10-12 days after surgery. Mainly Gram-positive microorganisms of endogenous breast flora are involved, such as coagulase-negative staphylococci, Staphylococcus aureus, methicillin-resistant $S$. aureus (MSRA), Streptococcus pyogenes, Propionibacterium acnes, diphtheroids, lactobacilli, or Bacillus species, but rare pathogens may also be involved. The endogenous breast flora can reach deeper tissues through breast ducts or during surgery. According to different studies [11,12], the microorganism most 
frequently observed is coagulase-negative staphylococci but in a recent single retrospective review [28], 67\% of implant infections were due to $S$. aureus, with methicillin resistance in $68 \%$ of these cases. In the same study, Gram-negative bacteria were reported in only $6 \%$ of infections. Typically, the clinical features include marked breast erythema, edema, warmth with simultaneous onset of rapidly evolving pain, deformation, and occasionally dehiscence of the surgical wound. Fever and purulent drainage are not always present. Leukocytosis is often found, but it does not represent a specific marker. The patient may have nausea, vomiting, watery diarrhea, myalgia, lethargy, and skin rash. In particular, these latter symptoms are indicative of toxic shock syndrome (TSS), a life-threatening complication caused by toxin-producing strains of $S$. aureus and $S$. pyogenes [29,30]. TSS occurs within 12-24 hours after breast implant introduction, much earlier than usual surgical site infections [29]. Even reconstruction with autologous flaps is not riskless, as demonstrated by a case report that described a TSS following a DIEP-flap [31]. TSS is characterized by poor local signs along with systemic involvement: fever exceeding $38.9^{\circ} \mathrm{C}$, hypotension, macular erythroderma, nausea, vomiting, diarrhea, myalgia, lethargy, respiratory distress syndrome, coagulopathy, and rapidly evolving multi-organ failure. For systemic involvement, it is lifesaving to recognize TSS immediately, remove the prosthesis, and quickly start intravenous antibiotic therapy.

\section{Late infections}

The onset time of late infection extends from a few months to several years after breast implant introduction. The onset time of infections is different for saline implants and silicone implants: saline implant infections occur within 8 weeks (on average 4 weeks), while silicone implant infections occur within 26 weeks (on average 13 weeks) [27]. The earlier onset of saline implant infections may be linked to contamination during implant filling [28]. After augmentation mammaplasty, $0.8 \%$ of patients are affected by late infections. Late infection usually results from bacteremia and secondary colonization of prosthesis, also bilaterally [15]. As a result, any potential bacterial infection, even in distant places, may represent a risk factor and should be recognized as early as possible to start a systemic antibiotic treatment. Moreover, invasive diagnostic procedures and surgery in patients with breast implants should be associated with antibiotic prophylaxis. The lack of attention to asepsis measures in invasive procedures or surgery is another common risk factor for late infections. The microorganisms involved can be both Gram-positive and Gram-negative, but coagulasenegative staphylococci and Propionibacterium acnes are the most common [32]. The symptoms of late infections are less pronounced than those of acute infections: delayed wound healing, not marked breast pain, mildly erythematous skin, slightly warm and stretched skin, drainage not always present and not purulent, and prosthesis dislocation. In some cases, infection occurs only with general discomfort and fatigue. Another major clinical problem is capsular contracture caused by chronic inflammation, often linked to biofilm formation on the implant surface [32]. Biofilm is a sessile community of microorganisms, in which cells adhere irreversibly to each other on a foreign body surface and produce a polymeric matrix, made of polysaccharides and glycoproteins. Biofilm is related to antibiotic resistance in many subclinical infections. Biofilm stimulates inflammation, and as inflammation goes on, the risk of capsular contracture increases [33]. The main strategy to prevent biofilm formation is the application of accurate asepsis protocol. However, new techniques have been developed to avoid it: steroid therapy and intraluminal antibiotic therapy [33]. Jacombs et al. [34] performed a study on a pig model, using a circular antibiotic-impregnated mesh located under the prosthesis during surgery. Patients with medicated implants developed only a mild or moderate contracture (Baker I/II) as late as a year following the surgery, and the implant surface showed a single layer of microorganisms or isolated bacteria under an electron microscope.

\section{Rare infections}

Rare infections with acute or delayed onset can be caused by atypical mycobacteria commonly present in soil, in municipal tap water, and hospital water systems [35]. The contamination of water may be greater in developing countries due to the lack of microbiological checks. A survey of 2,062 augmentation mammaplasties reported an incidence rate of $0.013 \%$ for atypical mycobacteria infections [36]. However, the real incidence of these infections could be much greater because mycobacterial cultures are not commonly requested. For atypical mycobacteria, the onset time ranges from two weeks to over one year [37]. The non-pigmented, rapidly growing mycobacterium (RGM) is the most frequently isolated microorganism; up to $60 \%-80 \%$ of $\mathrm{RGM}$ 
infections are caused by $M$. fortuitum group, comprising $M$. chelonae, $M$. abscessus, and $M$. immunogenum. In developing countries, the reports of prosthetic RGM infections are on the rise [37]. Clinical features are local erythema and swelling and tension as in acute infections caused by Gram-positive bacteria, but without systemic involvement. Instead, uncommon late infections are due to Streptomyces [38], Klebsiella pneumoniae [39], Pasteurella multocida [40], Brucella [41], Listeria [42], Clostridium perfringens [43], Granulicatella adiacens [44], Enterococcus avium [45], Bacteroides fragilis [46], and Serratia marcescens [47]. Also, fungal infections are unusual; they generally occur late and mainly affect immunocompromised patients. Fungal infections by Trichosporon, Aspergillus flavus and Aspergillus niger, Candida albicans, Curvularia, Penicillium, and Paecilomyces variotii have all been described [48-50]. According to Saray et al. [51], the silicone membrane of saline-filled devices is impermeable to Candida and Aspergillus, but both fungi are able to spread from outside to inside through the injection port.

\section{Diagnosis and management}

The clinical features of breast implant infection are not constant. In the literature, both the importance and the non-specificity of the clinical framework are highlighted [51]. Patients often complain of discomfort and tension at the site of implantation, but these symptoms are often linked to hydrohematic periprosthetic fluid. Fever is generally found, but it is not always present [15]. Pain and erythema are present in a high percentage of patients. An ultrasound scan is able to show periprosthetic fluid in order to identify the size and location of the infection and its relationship with the implant. However, an ultrasound scan does not show whether the periprosthetic fluid is infectious or not [52]. The ultrasound has an important role in the ultrasound-guided drainage, while other imaging techniques have a limited role. All diagnostic and therapeutic options aim to eradicate infection and rescue the implant. This does not always happen, particularly in late infections and rare infections. The gold standard for diagnosis and identification of antibiotic resistance is bacterial culture with antibiogram using aspirated periprosthetic fluids or bioptic samples [52]. Blood culture in patients with suspected bacteremia is another useful method [52]. Cytological and immunohistochemical evaluations are necessary in unclear diagnostic cases, which raise the problem of differential diagnosis between subclinical infection and malignancies such as anaplastic large cell lymphoma [52]. The most frequent infections are caused by bacteria of endogenous skin flora, particularly coagulase-negative staphylococci and $S$. aureus [28]. Before culture and antibiogram, empiric therapy with vancomycin is recommended, based on the high number of infections due to beta-lactam resistant pathogens, among which are methicillinresistant $S$. aureus and coagulase-negative staphylococci. While waiting for culture results, therapy should include extended-spectrum cephalosporins or penicillins to act also on Gramnegative pathogens [52]. A lack of improvement following a prolonged empiric therapy and implant removal should raise suspicion of late or rare infections. Culture and antibiogram are important in identifying the pathogen to set the target therapy aimed at eradicating the infection. If fluid aspirate is negative in routine culture, a therapy must be carried out for two weeks in order to eliminate pathogens not identified, and an atypical mycobacterial culture must be taken into account. If the patient's condition worsens or does not improve within two days, it may be necessary to remove the implant and microbiologically analyze the material removed. Usually, patients with systemic infections and poor general conditions require implant removal. The removed implant must be analyzed for aerobic bacteria, anaerobic bacteria, mycobacteria, and fungi [52]. Capsulotomy is not mandatory, but is usually done. After prosthesis removal, systemic antibiotics should be given for 10-14 days [53], orally in less serious cases and intravenously in severe infections. Immediate reimplantation is not advised, and the best time to perform the reimplantation will depend on the pathogen detected and the length of antimicrobial therapy necessary to obtain the eradication. Reimplantation is often delayed up to three or six months, but there are no trials identifying optimal timing. In selected cases, after pocket washing with saline solution and betadine, the immediate reimplantation of new prostheses has been successful [54]. The necessity of removing the controlateral implant is also a matter of debate.

\section{Antibiotic prophylaxis}

The use of prosthesis for breast aesthetic and reconstructive surgery makes antibiotic prophylaxis necessary, according to the guidelines for prevention of surgical site infection provided by United States Centers for Disease Control and Prevention (CDC) [24]. Several studies support preoperative antibiotic 
prophylaxis to reduce the risk of surgical site infection (surgical site infection average rates of $14.4 \%$ in surgery without preoperative antibiotic prophylaxis versus $5.8 \%$ in surgery with preoperative antibiotic prophylaxis) [25]. The guidelines recommend a single dose of intravenous first- or second-generation cephalosporin before starting anesthetic procedures. Surgical time exceeding three hours may require an intraoperative dose of antibiotic. In patients with allergies to beta-lactam antibiotics, a non-beta-lactam antibiotic with adequate spectrum is recommended, such as clindamycin or cotrimoxazole. Brand [15], in his extensive survey of a group of 73 plastic surgeons, showed that preoperative antibiotic prophylaxis, irrigation of surgical pocket, and immersion of implants in a antiseptic or an antibiotic solution (e.g., cephalosporins, bacitracin, neosporin), are usually performed in surgical practice. A recent systematic review underlines the fact that antibiotic prophylaxis in reconstructive surgery lowers the risk of surgical site infection; this study also emphasized that continuing prophylaxis beyond 24 hours after surgery [25] may not be useful, which has already been suggested by the CDC. Some studies [26] suggest the use of medicated implants to achieve reduction of capsular contracture, but further prospective studies are needed before recommending a widespread use of medicated implants that may increase the selection of antibiotic-resistant bacterial strains.

\section{Conclusions}

Infections are significant complications of breast implants. The most common pathogens found are those residing on breast skin; this consideration is useful in directing empirical therapy. The clinical presentation does not always provide a clear framework, but the presence of fever and leukocytosis associated with edema and swelling should always suggest the possibility of infection. The frequent finding of multi-drug resistance emphasizes the importance of culture and antibiogram. More attention should be given to the possibility of atypical mycobacteria infections, which are on the rise in developing countries. Therefore, we recommend specific culture to detect the growth of mycobacteria. Taking immediate action is essential in order to avoid implant removal, especially in patients with late infections who requested medical counseling very late. Prosthetic infections can be subtle but also very aggressive and should be treated with great attention until they are fully eradicated. Strict asepsis protocol associated with preoperative antibiotic prophylaxis, irrigation of the surgical pocket, and implant immersion in an antibiotic solution can lower the risk of surgical site infection. These tips could be used to decrease the risk of infection also increase the number of women predisposed to breast plastic surgery and in particular to immediate breast reconstruction in developing countries. We do not recommend using reused expanders to bring down health care costs in developing countries due to the higher rate of complications with related care costs, as mentioned in several reports $[22,23]$.

\section{Acknowledgements}

The authors dedicate this paper to Francesco Farace, Assistant Professor in Plastic Surgery at the University of Sassari, who prematurely died in the summer of 2013. He was a friend and a prime example of a scientist and surgeon for all of us.

\section{References}

1. Kjøller K, Hölmich LR, Jacobsen PH, Friis S, Fryzek J, McLaughlin JK, Lipworth L, Henriksen TF, Jørgensen S, Bittmann S, Olsen JH (2002) Epidemiological investigation of local complications after cosmetic breast implant surgery in Denmark. Ann Plast Surg 48: 229-237.

2. Malavaud S, Reme C, Gangloff D, Roques C, Chavoin JP (2005) Surgical site infection surveillance in breast implants surgery. Ann Chir Plast Esthet 50: 134-137.

3. Castell v. Greef (1994) South Africa. Supreme Court, Cape Provincial Division. S Afr Law Rep 17: 408-410.

4. Alderman A, Wilkins E, Kim H, Lowery JC (2002) Complications in postmastectomy breast reconstruction: twoyear results of the Michigan Breast Reconstruction Outcome Study. Plast Reconstr Surg 109: 2265-2274.

5. Clegg HW, Bertagnoll P, Hightower AW, Baine WB (1983) Mammaplasty-associated mycobacterial infection: a survey of plastic surgeons. Plast Reconstr Surg 72: 165-169.

6. Pinsolle V, Grinfeder C, Mathoulin-Pelissier S, Faucher A (2006) Complications analysis of 266 immediate breast reconstructions. J Plast Reconstr Aesthet Surg 59: 1017-1024.

7. Kirkland KB, Briggs JB, Trivette SL, Wilkinson WE, Sexton J (1999) The impact of surgical-site infections in the 1990s: Attribut- able mortality, excess length of hospitalization, and extra costs. Infect Control Hosp Epidemiol 20: 725-730.

8. Olsen M, Chu-Ongsakul S, Brandt K, Dietz JR, Mayfield J, Fraser VJ (2008) Hospital-associated costs due to surgical site infection after breast surgery. Arch Surg 143: 53-60.

9. Reilly J, Twaddle S, McIntosh J, Kean L (2001) An economic analysis of surgical wound infection. J Hosp Infect 49: 245 249.

10. Shameem H, Yip CH, Fong E (2008) Immediate breast reconstruction after mastectomy-why do women choose this option? Asian Pac J Cancer Prev 9: 409-412.

11. Francis SH, Ruberg RL, Stevenson KB, Beck CE, Ruppert AS, Harper JT, Boehmler JH, Miller MJ (2009) Independent risk factors for infection in tissue expander breast reconstruction. Plast Reconstr Surg 124: 1790-1796. 
12. Nahabedian MY, Tsangaris T, Momen B, Manson P (2003) Infectious complications following breast reconstruction with expanders and implants. Plast Reconstr Surg 112: 467-476.

13. Araco A, Gravante G, Araco F, Delogu D, Cervelli V, Walgenbach K (2007) Infections of breast implants in aesthetic breast augmentations: a single-center review of 3002 patients. Aesthetic Plast Surg 31: 325-329.

14. McCarthy C, Disa J, Pusic A, Mehrara BJ, Cordeiro PG (2008) The effect of closed-suction drains on the incidence of local wound complications following tissue expander/implant reconstruction: a cohort study. Plast Reconstr Surg 119: 20182022.

15. Brand KG (1993) Infection of mammary prostheses: a survey and the question of prevention. Ann Plast Surg 30: 289-295.

16. Liu A, Kao H, Reish R Hergrueter CA, May JW Jr, Guo L (2011) Post-operative complications in prosthesis-based breast reconstruction using acellular dermal matrix. Plast Reconstr Surg 127: 1755-1762.

17. Olsen M, Lefta M, Dietz J, Brandt KE, Aft R, Matthews R, Mayfield J, Fraser VJ (2008) Risk factors for surgical site infection after major breast operation. J Am Coll Surg 207: 326-335.

18. Liang MD, Narayanan K, Ravilochan K, Roche K (1993) The permeability of tissue expanders to bacteria: an experimental study. Plast Reconstr Surg 92: 1294-1297.

19. Javaid M, Shibu M (1999) Breast implant infection following nipple piercing. Br J Plast Surg 52: 676-677.

20. Peled A, Itakura K, Foster R, Hamolsky D, Tanaka J, Ewing C, Alvarado M, Esserman LJ, Hwang ES (2010) Impact of chemotherapy on postoperative complications after mastectomy and immediate breast reconstruction. Arch Surg 145: 880-885.

21. Agrawal K (1993) The reuse of tissue expanders in developing countries. Plast Reconstr Surg 92: 372-373.

22. Anger J (1991) The reuse of tissue expanders in developing countries. Plast Reconstr Surg 88: 1114-5.

23. Westreich M, Yeschua $R$ (1995) The reuse of tissue expanders in developing countries. Plast Reconstr Surg 95: 200.

24. Mangram AJ, Horan TC, Pearson ML, Silver LC, Jarvis WR (1999) Guideline for prevention of surgical site infection, 1999. Hospital Infection Control Practices Advisory Committee. Infect Control Hosp Epidemiol 20: 250-278.

25. Phillips BT, Bishawi M, Dagum AB, Khan SU, Bui DT (2013) A systematic review of antibiotic use and infection in breast reconstruction: what is the evidence? Plast Reconstr Surg 131: 1-13.

26. Adams WP Jr, Conner WC, Barton FE Jr, Rohrich RJ (2001) Optimizing breast-pocket irrigation: the post-betadine era. Plast Reconstr Surg 107: 1596-1601.

27. Basile A, Basile F, Basile A (2005) Late infection following breast augmentation with textured silicone gel-filled implants. Aesthet Surg J 25: 249-254.

28. Feldman EM, Kontoyiannis DP, Sharabi SE, Lee E, Kaufman Y, Heller L (2010) Breast implant infections: is cefazolin enough? Plast Reconstr Surg 126: 779-785.

29. Holm C, Muhlbauer W (1998) Toxic shock syndrome in plastic surgery patients: case report and review of the literature. Aesthetic Plast Surg 22: 180-184.

30. Brown SL, Hefflin B, Woo EK, Parmentier CM (2001) Infections related to breast implants reported to the Food and Drug Administration, 1977-1997. J Long Term Eff Med Implants 11: 1-12.
31. Tare M, Durcan J, Niranjan N (2010) A case of toxic shock syndrome following a DIEP breast reconstruction. J Plast Reconstr Aesthet Surg 63: e261-262.

32. Rieger UM, Mesina J, Kalbermatten DF, Haug M, Frey HP, Pico R, Frei R, Pierer G, Lüscher NJ, Trampuz A (2013) Bacterial biofilms and capsular contracture in patients with breast implants. Br J Surg 100: 768-774.

33. Pajkos A, Deva AK, Vickery K, Cope C, Chang L, Cossart YE (2003) Detection of subclinical infection in significant breast implant capsules. Plast Reconstr Surg 111: 1605-1611.

34. Jacombs A, Allan J, Hu H, Valente PM, Wessels WL, Deva AK, Vickery K (2012) Prevention of Biofilm-Induced Capsular Contracture With Antibiotic-Impregnated Mesh in a Porcine Model. Aesthet Surg J 32: 886.

35. Vinh DC, Rendina A, Turner R, Embil JM (2006) Breast implant infection with Mycobacterium fortuitum group: report of case and review. J Infect 52: e63-67.

36. Clegg HW, Bertagnoll P, Hightower AW, Baine WB (1983) Mammaplasty-associated mycobacterial infection: a survey of plastic surgeons. Plast Reconstr Surg 72: 165-169.

37. Thomas M, D'Silva JA, Borole AJ, Chilgar RM. (2013) Periprosthetic atypical mycobacterial infection in breast implants: a new kid on the block! J Plast Reconstr Aesthet Surg. 66: e16-19.

38. Manteca A, Palaez A, del Mar Garcia-Suarez M, Hidalgo E, Lopez S, Mendez FJ (2009) A rare case of silicone mammary implant infection by Streptomyces spp. in a patient with breast reconstruction after mastectomy: taxonomic characterization using molecular techniques. Diagn Microbiol Infect 63: 390-393.

39. Bernardi C, Saccomanno F (1998) Late Klebsiella pneumoniae infection following breast augmentation: case report. Aesthetic Plast Surg 22: 222-224.

40. Mathieu D, Rodriguez H, Jacobs F (2008) Breast prosthesis infected by Pasteurella multocida. Acta Clin Belg 63: 351.

41. De B, Stauffer L, Koylass M, Sharp SE, Gee JE, Helsel LO, Steigerwalt AG, Vega R, Clark TA, Daneshvar MI, Wilkins PP, Whatmore AM (2008) Novel Brucella strain (BO1) associated with a prosthetic breast implant infection. J Clin Microbiol 46: 43-49.

42. Gnanadesigan N, Pechter EA, Mascola L (1994) Listeria infection of silicone breast implant. Plast Reconstr Surg 94: 531-533, discussion 534-535.

43. Hunter J, Padilla M, Cooper-Vastola S (1996) Late Clostridium perfringens breast implant infection after dental treatment. Ann Plast Surg 36: 309-312.

44. Del Pozo J, Garcia-Quetglas E, Henaez S, Serrera A, Alonso M, Pina L, Leiva J, Azanza JR (2008) Granulicatella adiacens breast implant-associated infection. Diagn Microbiol Infect Dis 61: 58-60.

45. Ablaza VJ, LaTrenta GS (1998) Late infection of a breast prosthesis with Enterococcus avium. Plast Reconstr Surg 102: 227-230.

46. Petit F, Maladry D, Werther JR, Mitz V (1998) Late infection of breast implant, complication of colonic perforation. Review of the literature. Role of preventive treatment. Ann Chir Plast Esthet 43: 559-562.

47. Pegues D, Shireley L, Riddle C, Anderson RL, Vess RW, Hill BC, Jarvis WR (1991) Serratia marcescens surgical wound infection following breast reconstruction. Am J Med 91: 173S-178S. 
48. Reddy BT, Torres HA, Kontoyiannis DP (2002) Breast implant infection caused by Trichosporon beigelii. Scand J Infect 34: 143-144.

49. Tian HH, Tan SM, Tay KH (2007) Delayed fungal infection following augmentation mammoplasty in an immunocompetent host. Singapore Med J 48: 256-258.

50. Coady MSE, Gaylor J, Knight SL (1995) Fungal growth within a silicone tissue expander: case report. Br J Plast Surg 48: $428-430$

51. Saray A, Kilic D, Kaygusuz S, Boyunaga H, Ozlük O (2004) Fungal growth inside saline-filled implants and the role of injection ports in fungal translocation: in vitro study. Plast Reconstr Surg 114: 1170-1178.

52. Washer L, Gutowsky L (2012) Breast implant infections. Infect Dis Clin North Am 26: 111-125.

53. Darouiche RO (2004) Treatment of infections associated with surgical implants. N Engl J Med 350: 1422-1429.
54. Spear SL, Howard MA, Boehmler JH, Ducic I, Low M, Abbruzzesse MR (2004) The infected or exposed breast implant: management and treatment strategies. Plast Reconstr Surg 113: 1634-1644.

\section{Corresponding author}

Domenico Pagliara

Via Eugenio Siciliano 39, I-84014 Nocera Inferiore

Salerno, Italy

Phone: +39 3935683365

E-mail: domenicopag@gmail.com

Conflict of interests: No conflict of interests is declared. 